\title{
In-Situ Fabrication of Expanded Graphite-Carbon Nanotube Nanocomposite With Enhanced Thermal Conductivity
}

\author{
Jun Cao ${ }^{1,2}$, Chuanling Men ${ }^{1}$, Hongyuan $\mathrm{Chen}^{2}$, Geng $\mathrm{Xu}^{2}$, Yongyi Zhang ${ }^{2}$ \& Qingwen $\mathrm{Li}^{2}$ \\ ${ }^{1}$ School of Energy and Power Engineering, University of Shanghai for Science and Technology, Shanghai, \\ China \\ ${ }^{2}$ Suzhou Institute of Nano-tech and Nano-bionics, Chinese Academy of Sciences, Suzhou, China \\ Correspondence: Yongyi Zhang, Suzhou Institute of Nano-tech and Nano-bionics, Chinese Academy of Sciences, \\ Suzhou 215123, China. Tel: 86-512-6287-2764. E-mail: yyzhang2011@sinano.ac.cn
}

Received: October 22, 2013 Accepted: November 11, 2013 Online Published: November 21, 2013

doi:10.5539/jmsr.v3n1p50 URL: http://dx.doi.org/10.5539/jmsr.v3n1p50

\begin{abstract}
The three-dimensional (3D) expanded graphite-carbon nanotube (EG-CNT) nanocomposite was prepared by in situ growing CNTs between graphite layers in thermally expanded graphite. The morphology and microstructure of the nanocomoposite were characterized by SEM, TEM, Raman and XRD analyses, respectively. The results show that intimately contacted EG-CNT interface has been formed within the multilayered composite architecture. Furthermore, paraffin was used as a filler to occupy the free spaces of the EG-CNT nanocomposite for thermal application. The as-obtained composite structure is inclined to conduct heat isotropically and shows a thermal conductivity higher than $10 \mathrm{~W} / \mathrm{m} \cdot \mathrm{K}$ at a paraffin loading of $49 \mathrm{wt} . \%$, which indicates that EG-CNT nanocomposite could be promising candidates for thermal management in electronic package.
\end{abstract}

Keywords: expanded graphite, carbon nanotube, multilayer structure, nanocomposites, thermal properties

\section{Introduction}

Highly thermal conductive carbon/carbon composite materials are usually based on graphite material due to its ultra-high thermal conductivity in in-plane directions of graphene layer (Fukushima, Drzal, Rook, \& Rich, 2006; Klemens \& Pedraza, 1994). Whilst carbon nanomaterials, such as carbon nanotubes (CNTs) and graphene, which are usually used as the thermally conductive fillers (Huang, Liu, Wu, \& Fan, 2005; Moisala, Li, Kinloch, \& Windle, 2006; Yu et al., 2008), could largely enhance the thermal conductivities of the resulting nanocomposites owing to their strong direction-dependent thermal transport properties (Balandin et al., 2008; Balandin, 2011; Berber, Kwon, \& Tománek, 2000). For example, individual carbon nanotube (CNT) shows a thermal conductivity higher than $3000 \mathrm{~W} / \mathrm{mK}$ and could be more than $6000 \mathrm{~W} / \mathrm{mK}$ for individual single-walled CNTs. The in-plane thermal conductivity of graphene could be higher than $5000 \mathrm{~W} / \mathrm{mK}$. However, the degree of the enhancement has been limited by the size of these nanomaterials and the large thermal resistance among these nano-sized building blocks (Cai et al., 2010; Ghosh et al., 2008). Furthermore, their anisotropy in thermal conductivity (Xu, Chen, Gu, \& Duan, 2009) has made it necessary to construct an effectively thermal conductive network with isotropy in the nanocomposites.

Expanded graphite (EG), a three-dimensional plate-like nanostructure with a higher volume expansion ratio than that of the regular graphite, has been widely used as the thermal conductive material due to its excellent properties, such as compatibility, spring-back, flexibility, heat conducting, anti-acid, anti-base and so on (Chen, Wu, Weng, Wu, \& Yan, 2003; Tang, Wu, Sun, \& Fang, 2009). However, both one-dimensional CNTs and there-dimensional EG exhibited direction-dependent thermal conductivities, which makes them difficult to be used as the thermal management materials with the aim of constructing three-dimensional thermally conductive networks in the composites. By introducing CNTs into EG, the EG-CNT nanocomposite is expected to be with excellent performance for heat transportation. However, the as-prepared CNTs could not be easily inserted into the interspace among graphene layers in EG. This issue has largely affected the construction of three-dimensional nanocomposites based on CNT and EG.

In this manuscript, we report the synthesis of an EG-CNT hybrid nanocomposite and its thermal conductive performance fitted with paraffin. Multilayered EG-CNT composite nanostructures were prepared by an in situ 
chemical vapor deposition (CVD) process (Du et al., 2011; Fan et al., 2010). In this way, neighboring graphite sheets were separated by numerous CNTs, which were rooted in and stabilized by the EG substrate, so the CNTs aggregation was also hindered. In comparison with the pure EG materials, the thermal connectivity of the resulting EG-CNT nanocomposite along vertical direction was improved by bridging graphite sheets with CNT materials and this nanocomposite power could be inclined to conduct heat isotropically. Furthermore, paraffin was used as a matrix to embed EG-CNT nanocomposite and the resulting material shows a thermal conductivity higher than $10 \mathrm{~W} / \mathrm{m} \cdot \mathrm{K}$, which indicates it a promising candidate for thermal management in electronic package.

\section{Method}

\subsection{Materials}

Expansible graphite (CX-200) was purchased from Qingdao Tianheda Graphite Co., Ltd., Qingdao, $\mathrm{China}^{\mathrm{NiCl}} \mathrm{N}_{2}$ and paraffin were purchased from Aladdin Reagent Co., Ltd., Shanghai, China.

Figure 1 illustrates our schematic procedure of preparing EG-CNT hybrid nanomaterials.
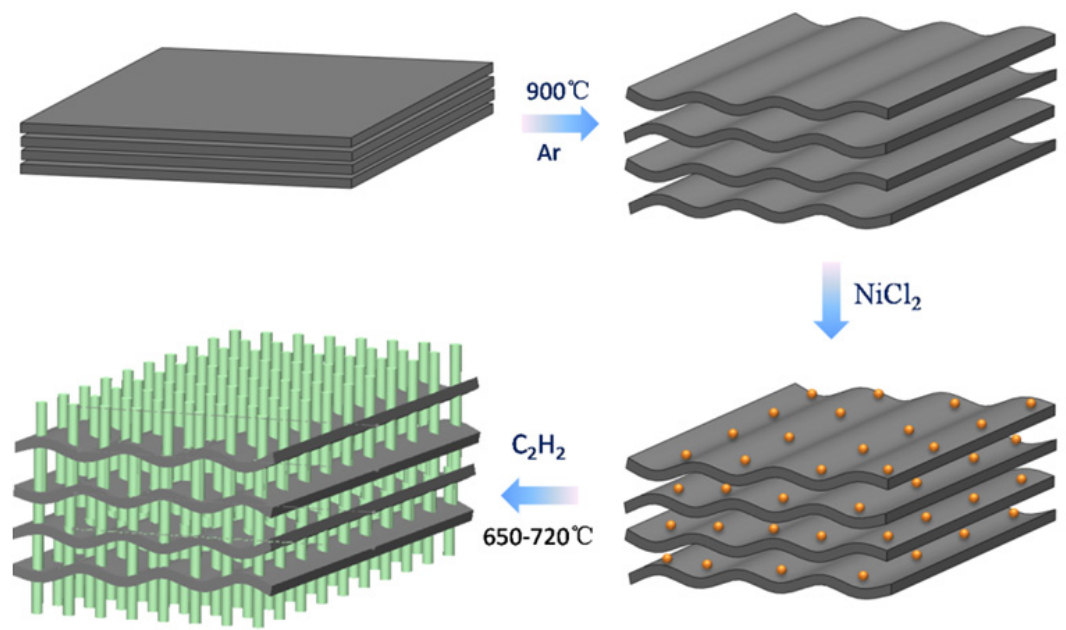

Figure 1. Schematic illustration of the procedure for the preparation of 3D EG-CNT nanocomposite, showing stacked layers of expansible graphite, the loose structures of EG, catalyst particles adhered onto layer surface and CNTs in between EG layers after growth

\subsection{Preparation of EG-NiCl ${ }_{2}$ Composites}

Firstly, the purchased expansible graphite was put into a tube furnace and heated up to $850-900{ }^{\circ} \mathrm{C}$ under a purging gas of argon $(500 \mathrm{sccm})$ to get EG. Then the as-prepared EG was immerged into a homogenous $\mathrm{NiCl}_{2}$-ethanol solution $(0.02 \mathrm{mg} / \mathrm{ml})$ for $2 \mathrm{~h}$ to adsorb $\mathrm{Ni}$ ions as the catalyst of CNT growth and then dried at 60 ${ }^{\circ} \mathrm{C}$ under vacuum for $12 \mathrm{~h}$ to remove the moisture content.

\subsection{Fabrication of $3 D$ EG-CNT Composite Architecture}

EG- $\mathrm{NiCl}_{2}$ was put into the center of furnace and heated to $650-720{ }^{\circ} \mathrm{C}$ under a gas mixture flow of $\mathrm{Ar}(1600$ $\mathrm{sccm})$ and $\mathrm{H}_{2}(400 \mathrm{sccm})$. When the furnace reaches the preset temperature, $\mathrm{C}_{2} \mathrm{H}_{2}$ was introduced as the carbon source with a flow rate of $180 \mathrm{sccm}$. The growth time was 10 15 min.

\subsection{Fabrication of EG-CNTs Thermal Conductive Mats}

The as-prepared EG-CNT nanocomposites were mixed with different loading of paraffin and then stirred uniformly. The composites were then heated to $60{ }^{\circ} \mathrm{C}$ with continuously stirring until a uniform paste was formed. The as-formed composites was cooled down to room temperature and made to mats by hydraulic press.

\subsection{Characterization}

The morphology of the EG-CNT nanocomposite was characterized by scanning electron microscopy (SEM, Hitachi S4800) and transmission electron microscope (TEM, FEI Tecnai G2 F20 S-Twin), The phase composition was characterized by X-ray diffraction (XRD, D8 Advance, Bruker AXS) with $\mathrm{Cu} \mathrm{K}_{a}$ radiation generator $(0.151 \mathrm{~nm}, 40 \mathrm{kV}, 40 \mathrm{~mA})$ and Raman spectroscopy with a Renishaw RM3000 Raman microtoscope (laser: $514 \mathrm{~nm}$ with a $2 \mu \mathrm{m}$ spot size). Porosity was measured by a method reported (Kohlmeyer, Lor, Denga, 
Liub, \& Chena, 2011). The thermal conductivity was measured by a DRL-III thermal conductivity meter (Xiangtan City Instrument \& Meter Co., Ltd., Xiangtan, China) under a pressure of 40 psi, which allowed an efficient contact between the sample and substrates and had negligible influences on the structure (Xing et al., 2013).

\section{Results and Discussion}

\subsection{The Microstructure of Samples}

The resultant EG-CNT material showed a several fold increase in mass (4 6 times) with a concomitant volume expansion of a few hundred times with respect to the original expansible graphite.

The SEM images of the hybrid structure are shown in Figure 2. Figures 2(a-f) show the original expansible graphite, EG and CNT-EG hybrid structure, respectively. The difference of the samples in microstructure can be clearly observed. The untreated expansible graphite displays typical multilayered structure (Figure 2a). High temperature would induce thermal expansion of oxygen-containing groups trapped between graphite layers and lead a dramatic expansion of graphite layers (Chen et al., 2003; Stankovich et al., 2007), so the loose structure can be clearly observed on the as-prepared EG after rapid expansion at a high temperture (Figure 2b). Figure 2c suggests the thickness of the exfoliated graphite nanoflakes of the porous structure is less than $30 \mathrm{~nm}$. After immerging of the catalyst precursor and subsequent in situ growth process, CNTs were intercalated into the gaps among the thermally expanded graphite layers, the formed EG-CNT "bush" are clearly seen in Figure 2d under a higher magnification. It also shows that the CNTs grew from both sides of neighboring graphite sheets, with an intimately contacted EG-CNT interface. The diameter of the as-grown CNTs was not uniform and the tube length is up to $10 \mu \mathrm{m}$ (Figure 2d). TEM observation further shows that the as-grown CNTs are mainly multi-walled CNTs (MWCNTs) with a diameter of 6-25 nm and 2-12 concentric walls (Figure 2f). Interestingly, there is a massive growth of MWCNTs on and between graphite layers, so the bush of EG-CNT nanocomposite looks like a carpenter worm (Figure 2e).
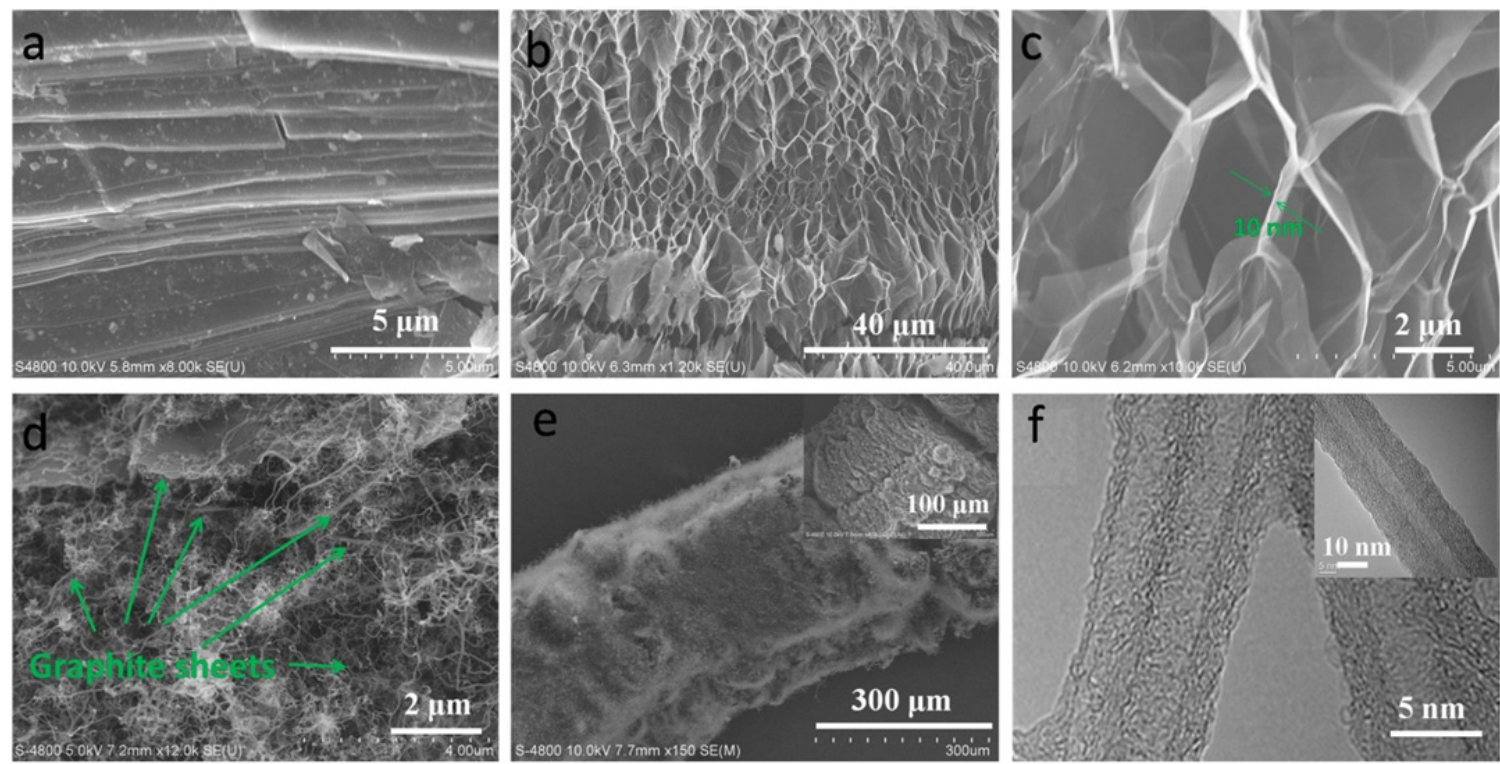

Figure 2. SEM images of (a) the pristine expandsible graphite and the thermally expanded EG (b) and (c).

Typical SEM images of the 3D EG-CNTs composites under different magnifications. (d, e). (f)TEM of a typical

in situ growed CNT with 10-25(insert) walls. (h)The macroscopic morphology of the as-obtained EG-CNTs composites

\subsection{XRD and Raman Analysis}

XRD was used to investigate the crystal structure of the nanocomposite. Figure $3 \mathrm{a}$ shows two sharp peaks at $2 \theta$ $=26.5^{\circ}$ and $56.1^{\circ}$, which were correspond to the (002) and (004) lattice plane of graphite, respectively (Shi, Barker, Saïdi, \& Koksbang, 1996; Welham \& Williams, 1998). The thermal treatment caused a significant intensity decrease for the (002) and (004) peaks, which was accompanied by the appearance of a new broadband centered at $2 \theta=8.5^{\circ}$. These changes imply the presence of expanded stacking regions of more disordered thinner graphite sheets (Du et al., 2011; Fan et al., 2010). As shown in Figure 3a of the contrastive curve of the EG-CNT 
nanocomposite, the (004) peak has a significant intensity decrease and is almost disappear. The (002) characteristic peak of CNTs appears at $2 \theta=25.3^{\circ}$. Owing to the reduction of $\mathrm{H}_{2}$ during the CNT growing process, the Ni was got as catalyst, which was demonstrated by the (111) and (200) characteristic peaks of $\mathrm{Ni}$ at $2 \theta=44.5^{\circ}$ and $2 \theta=52^{\circ}$.

The structure changes caused by the thermal expansion and CNTs intercalation were further analyzed by Raman spectra (Figure 3b). The pure expansible graphite has a characteristic peak at $1582 \mathrm{~cm}^{-1}$ (G peak) (Ferrari et al., 2006; Tuinstra \& Koenig, 1970) and the EG has a new peak at $1355 \mathrm{~cm}^{-1}$ (D peak), which reflect the thermal treatment-induced disordered structures (Du et al., 2011). The $\mathrm{I}_{\mathrm{D}} / \mathrm{I}_{\mathrm{G}}$ ratio increases with the CNT grown due to the increased disordered graphite sheets and existence of the CNTs during the process of CNTs growing.
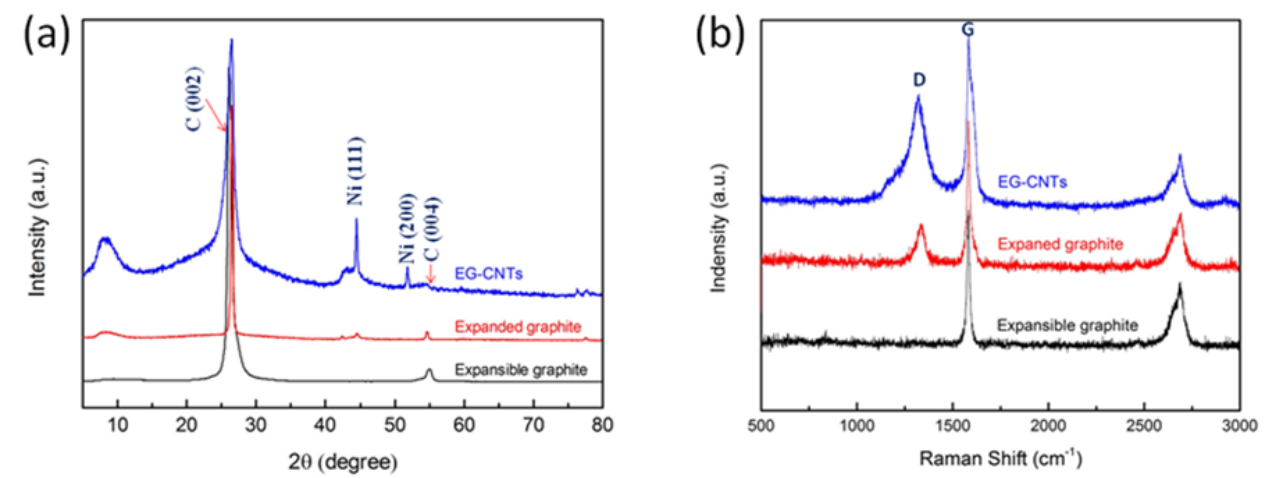

Figure 3. (a)XRD patterns and (b) Raman spectra for the expendsible graphite, EG and the as-prepared EG-CNT

\subsection{The Enhanced Thermal Conductivity}

nanocomposite

Paraffin was used to fill into the EG-CNT nanocomposite with the aim of preparing freestanding mats. Figure 4a shows the EG-CNT composite mats of EG-CNT nanocomposite with different paraffin loading. Although the thermal conductivity of paraffin $(0.21 \mathrm{~W} / \mathrm{m} \cdot \mathrm{K})$ is much less than that of carbon materials, it is an excellent phase change material and can fill the empty spaces of the composites to reduce thermal resistance (Kim \& Drzal, 2009; Marín, Zalba, Cabezab, \& Mehlingc, 2005; Py, Olives, \& Mauran, 2001), as shown in the schematic diagram of Figure $4 b$.
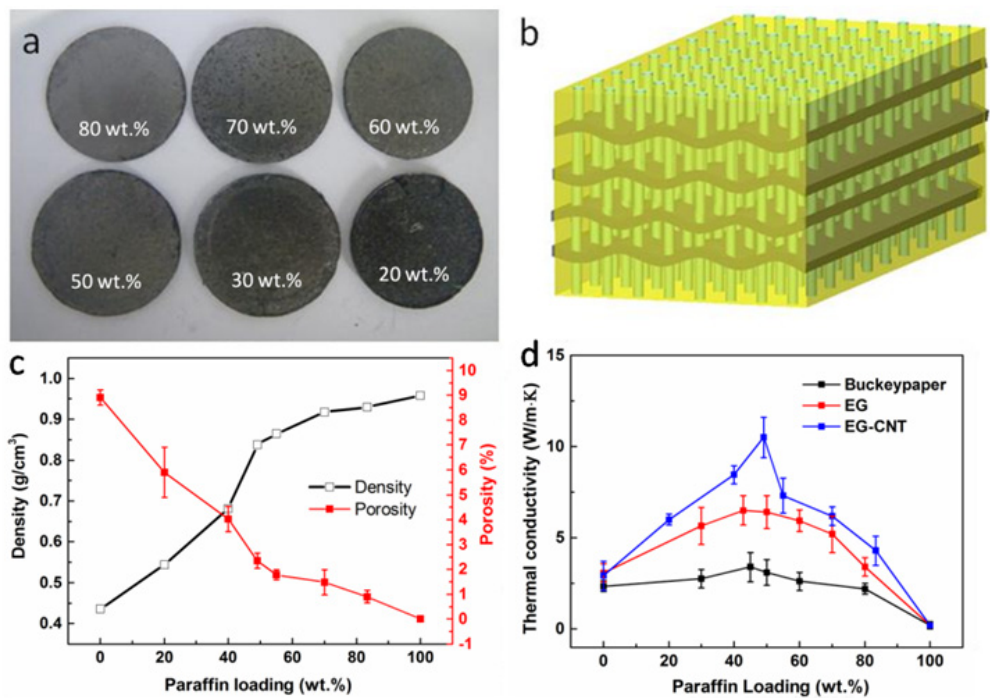

Figure 4. (a)Photograph of the as-prepared EG-CNT composite mats with different paraffin loading of 80, 70, 60, 50, 30, 20 wt.\%. (b) Schematic diagram of EG-CNT nanocomposites filled empty spaces with paraffin. (c) Variation of density and porosity of the composite with the paraffin content (d) Thermal conductivity of buckypaper, EG and EG-CNT nanocomposite as functions of paraffin content 
In order to demonstrate the enhanced thermal conductivity of the EG-CNT nanocomposite, we made the contrastive measurement of relevant materials-buckypaper, EG and EG-CNT nanocomposite. The thermal conductivities of different samples with different paraffin loading had been tested. As shown in Figure $4 \mathrm{~d}$, there is a regular and monotonic increase for the thermal conductivities of these specimens at the beginning, following by the gradual increase with the increase of the paraffin content. It could be attributed to the gradually decrease of the empty spaces in the EG-CNT nanocomposites (Figure 4c). When the paraffin loading amount was at 49 wt.\%, the thermal conductivity of the EG-CNT nanocomposites increases to $10.5 \mathrm{~W} / \mathrm{m} \cdot \mathrm{K}$, while at higher loadings, the paraffin has already fully filled the empty spaces and the extra paraffin only coated the nanocomposites, which leads to the decrease of their thermal conductivity. As a result, it accords the variation trend of the curve. Another two samples accord with the same variation trend (Figure 4d). Meanwhile, direction-dependent thermal transport properties limited a further improved thermal conductivities of buckypapers and EG, so the EG-CNT composites inclined to thermal conductive isotropy obviously has higher thermal conductivity than that of another materials(respectively buckeypaper, $3.4 \mathrm{~W} / \mathrm{m} \cdot \mathrm{K}$ at $45 \mathrm{wt} . \%$ loading and $\mathrm{EG}, 6.5 \mathrm{~W} / \mathrm{m} \cdot \mathrm{K}$, at $42.8 \mathrm{wt} . \%$ loading).

The EG-CNT nanocomposite filled with paraffin has a compact sandwich structure upon stacking, which ensured the EG-CNT composite mats excellent thermal and electrical conductive performance. Figure 5(a) shows the as-prepared EG-CNT composite mat with a paraffin loading of 49 wt.\%. As shown in Figure 5(b) and (c), the composite mat has a intimately contacted EG-CNT interface with paraffin. The filling of paraffin resulted in significant changes of the electrical and thermal properties. As shown in Figure 5(d), the electrical conductivities along in-plane and perpendicular directions $\left(\sigma_{/}, \sigma_{\perp}\right)$ decreased with the increasing of paraffin loading. At the $49 \mathrm{wt} . \%$ of paraffin loading, the electrical conductivities remained $17 \mathrm{~S} / \mathrm{cm}(\sigma /)$ and $12 \mathrm{~S} / \mathrm{cm}(\sigma$ ${ }_{\perp}$ ). The isotropic conductive networks in the nanocomposite make the composite mats an isotropous conductivity, which was confirmed by the slight change of $\sigma / / \sigma_{\perp}$.
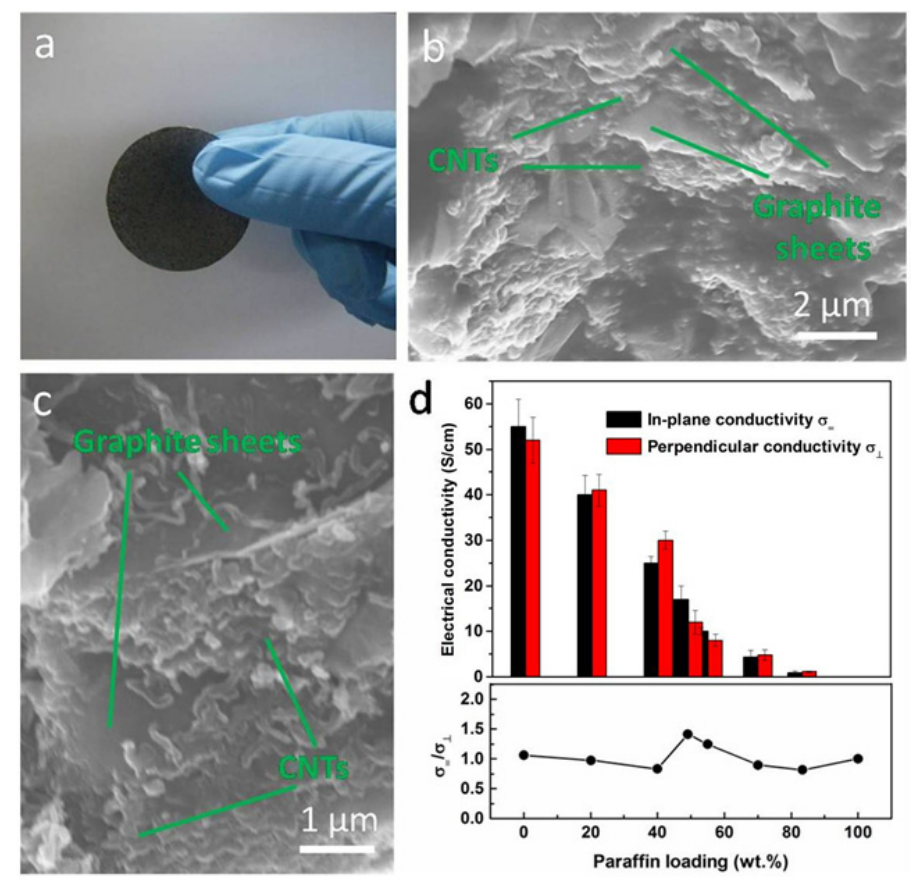

Figure 5. Photograph of the as-produced EG-CNT composite mat with a paraffin loading of $49 \mathrm{wt} . \%$ (a). The SEM image of a typical inner structure of EG-CNT composite mat filled with paraffin $(b, c)$. In-plane and perpendicular electrical conductivities (top panel) and the corresponding isotropy (bottom panel) for the

EG-CNT mats at different paraffin contents (d)

\section{Conclusions}

In summary, we have developed multilayered three-dimensional EG-CNT nanocomposite by an in situ CVD process. The EG-CNT nanocomposite exhibits multilayered architectures upon stacking and constructs a three-dimensional isotropous thermal conducting network. The EG-CNT composite mat filled with paraffin at 49 
wt.\% has a thermal conductivity of $10.5 \mathrm{~W} / \mathrm{m} \cdot \mathrm{K}$. The excellent thermal property of the nanocomposite indicates potential application in electronic package.

\section{Acknowledgements}

This work was supported by the Youth Innovation Promotion Association, CAS for Yongyi Zhang, and National Natural Science Foundation of China (No. 10834004, 51202282) and the Natural Science Foundation of Jiangsu Province (No. BK20131172).

\section{References}

Balandin, A. A. (2011). Thermal properties of graphene and nanostructured carbon materials. Nature Materials, 10(8), 569-581. http://dx.doi.org/10.1038/nmat3064

Balandin, A. A., Ghosh, S., Bao, W., Calizo, I., Teweldebrhan, D., Miao. F., \& Lau, C. N. (2008). Superior thermal conductivity of single-layer graphene. Nano Letters, 8(3), 902-907. http://dx.doi.org/10.1021/n10731872

Berber, S., Kwon, Y. K., \& Tománek, D. (2000). Unusually high thermal conductivity of carbon nanotubes. Physical Review Letters, 84(20), 4613. http://dx.doi.org/10.1103/PhysRevLett.84.4613

Cai, W., Moore, A. L., Zhu, Y., Li, X., Chen, S., Shi, L., \& Ruoff, R. S. (2010). Thermal transport in suspended and supported monolayer graphene grown by chemical vapor deposition. Nano Letters, 10(5), 1645-1651. http://dx.doi.org/10.1021/n19041966

Chen, G., Wu, C., Weng, W., Wu, D., \& Yan, W. (2003). Preparation of polystyrene/graphite nanosheet composite. Polymer, 44(6), 1781-1784. http://dx.doi.org/10.1016/S0032-3861(03)00050-8

Du, F., Yu, D., Dai, L., Ganguli, S., Varshney, V., \& Roy, A. K. (2011). Preparation of tunable 3D pillared carbon nanotube-graphene networks for high-performance capacitance. Chemistry of Materials, 23(21), 4810-4816. http://dx.doi.org/10.1021/cm2021214

Fan, Z., Yan, J., Zhi, L., Zhang, Q., Wei, T., Feng, J., ... Wei, F. (2010). A three-dimensional carbon nanotube/graphene sandwich and its application as electrode in supercapacitors. Advanced Materials, 22(33), 3723-3728. http://dx.doi.org/10.1002/adma.201001029

Ferrari, A. C., Meyer, J. C., Scardaci, V., Casiraghi, C., Lazzeri, M., Mauri, F., ... Geim, A. K. (2006). Raman spectrum of graphene and graphene layers. Physical Review Letters, 97(18), 187401. http://dx.doi.org/10.1103/PhysRevLett.97.187401

Fukushima, H., Drzal, L. T., Rook, B. P., \& Rich, M. J. (2006). Thermal conductivity of exfoliated graphite nanocomposites. Journal of Thermal Analysis and Calorimetry, 85(1), 235-238. http://dx.doi.org/10.1007/s10973-005-7344-x

Ghosh, S., Calizo, I., Teweldebrhan, D., Pokatilov, E. P., Nika, D. L., Balandin, A. A., ... Lau, C. N. (2008). Extremely high thermal conductivity of graphene: Prospects for thermal management applications in nanoelectronic circuits. Applied Physics Letters, 92, 151911. http://dx.doi.org/10.1063/1.2907977

Huang, H., Liu, C. H., Wu, Y., \& Fan, S. (2005). Aligned carbon nanotube composite films for thermal management. Advanced Materials, 17(13), 1652-1656. http://dx.doi.org/10.1002/adma.200500467

Kim, S. \& Drzal, L. T. (2009). High latent heat storage and high thermal conductive phase change materials using exfoliated graphite nanoplatelets. Solar Energy Materials and Solar Cells, 93(1), 136-142. http://dx.doi.org/10.1016/j.solmat.2008.09.010

Klemens, P., \& Pedraza, D. (1994). Thermal conductivity of graphite in the basal plane. Carbon, 32(4), 735-741. http://dx.doi.org/10.1016/0008-6223(94)90096-5

Kohlmeyer, R. R., Lora, M., Denga, G., Liub, H., \& Chena, J. (2011). Preparation of stable carbon nanotube aerogels with high electrical conductivity and porosity. Carbon, 49(7), 2352-2361. http://dx.doi.org/10.1016/j.carbon.2011.02.001

Marín, J. M., Zalbaa, B., Cabezab, L. F., \& Mehlingc, H. (2005). Improvement of a thermal energy storage using plates with paraffin-graphite composite. International Journal of Heat and Mass Transfer, 48(12), 2561-2570. http://dx.doi.org/10.1016/j.ijheatmasstransfer.2004.11.027

Moisala, A., Li, Q., Kinloch, I. A., \& Windle, A. H. (2006). Thermal and electrical conductivity of single-and multi-walled carbon nanotube-epoxy composites. Composites Science and Technology, 66(10), 1285-1288. http://dx.doi.org/10.1016/j.compscitech.2005.10.016 
Py, X., Olives, R., \& Mauran, S. (2001). Paraffin/porous-graphite-matrix composite as a high and constant power thermal storage material. International Journal of Heat and Mass Transfer, 44(14), 2727-2737. http://dx.doi.org/10.1016/S0017-9310(00)00309-4

Shi, H., Barker, J., Saïdi, M. Y., \& Koksbang, R. (1996). Structure and lithium intercalation properties of synthetic and natural graphite. Journal of the Electrochemical Society, 143(11), 3466-3472. http://dx.doi.org/10.1149/1.1837238

Stankovich, S., Dikin, D. A., Piner, R. D., Kohlhaas, K. A., Kleinhammes, A., Jia, Y., ... Ruoff, R. S. (2007). Synthesis of graphene-based nanosheets via chemical reduction of exfoliated graphite oxide. Carbon, 45(7), 1558-1565. http://dx.doi.org/10.1016/j.carbon.2007.02.034

Tang, Q., Wu, J., Sun, H., \& Fang, S. (2009). Crystallization degree change of expanded graphite by milling and annealing. Journal of Alloys and Compounds, 475(1-2), 429-433. http://dx.doi.org/10.1016/j.jallcom.2008.07.063

Tuinstra, F., \& Koenig, J. L. (1970). Raman spectrum of graphite. The Journal of Chemical Physics, $53,1126$. http://dx.doi.org/10.1063/1.1674108

Welham, N., \& Williams, J. (1998). Extended milling of graphite and activated carbon. Carbon, 36(9), 1309-1315. http://dx.doi.org/10.1016/S0008-6223(98)00111-0

Xing, Y., Zhang, X., Chen, H., Chen, M., \& Li, Q. (2013). Enhancing electrical and thermal conductivities of buckypapers by sticking copper nanowires. Carbon, 601-506. http://dx.doi.org/10.1016/j.carbon.2013.05.030

$\mathrm{Xu}$, Y., Chen, X., Gu, B. L., \& Duan, W. (2009). Intrinsic anisotropy of thermal conductance in graphene nanoribbons. Applied Physics Letters, 95(23), 233116-233116-233113. http://dx.doi.org/10.1063/1.3272678

Yu, A., Ramesh, P., Sun, X., Bekyarova, E., Itkis, M. E., \& Haddon, R. C. (2008). Enhanced thermal conductivity in a hybrid graphite nanoplatelet-carbon nanotube filler for epoxy composites. Advanced Materials, 20(24), 4740-4744. http://dx.doi.org/10.1002/adma.200800401

\section{Copyrights}

Copyright for this article is retained by the author(s), with first publication rights granted to the journal.

This is an open-access article distributed under the terms and conditions of the Creative Commons Attribution license (http://creativecommons.org/licenses/by/3.0/). 